Plant Production Science

http:/www.journals.zu.edu.eg/journalDisplay.aspx?Journalld=1\&queryType=Master

\title{
SOME FACTORS AFFECTING THE VALUE OF HYDROPONIC SPROUTED BARLEY FOR GREEN FODDER
}

\author{
Faisal I. Yousof ${ }^{*}$, El-Shimaa E.I. Mustafa and M.A.H. Megahed \\ Seed Tech. Res. Dept., Field Crops Res. Inst., Agric. Res. Cent., Egypt
}

\section{Received: 03/04/2018 ; Accepted: 20/06/2018}

\begin{abstract}
Hydroponic sprouted barley is recently become an applied approach to provide a green fodder crop, particularly in arid and semiarid region such as Egypt. The crop is produced from germinating seeds under controlled growing conditions for a short period. Laboratory experiments have been performed to determine the effect of some seed soaking treatments and germination temperature on the quantity and quality of green fodder crop. Various stimulants i.e. gibberellin solution and yeast extract were used .Seed germination was made at different temperatures. The results indicated that seed soaked in gibberellin solution $(12 \mathrm{ppm})$ and yeast extract $(3 \mathrm{~g} / \mathrm{l})$ and germination temperature at $35^{\circ} \mathrm{C}$ exceeded other seed treatments as to the most parameters of the quantity and quality of green fodder crop. The simple correlation coefficients of seed germination (\%), germination speed index, seedling length and seedling fresh weight towards green fodder yield were positive and significant $(0.939,0.897,0.892$ and 0.928$)$, respectively. The stepwise regression analysis revealed that the relative contribution for seed germination (\%), seedling length and seedling fresh weight towards green fodder yield was $83.7 \%$. The results of chemical analyses of green fodder crop such as dry matter (\%), ash (\%), organic matter (\%), crude protein (\%), neutral detergent fiber (NDF) (\%), acid detergent fiber (\%) (ADF), ether extract (\%) (EE) and non-fiber carbohydrate (\%) (NCF) were consisted with those of germination temperature and seedling characters. These findings suggested that soaking barley seed in gibberellin solution $(12 \mathrm{ppm})$ and yeast extract $(3 \mathrm{~g} / \mathrm{l})$ as a good ,simple and cheap treatment to get high quality and maximize sprouted fodder yield under different temperatures throughout the year.
\end{abstract}

Key words: Barley, seed soaking, germination temperature, sprouted green fodder.

\section{INTRODUCTION}

The production of fresh green sprouts of barley can be made in special growing rooms, regardless of the weather and at any time of the year. This includes germinating barley seeds in hydroponic system for about 7 to 10 days where the length of forage mat reaches $15-30 \mathrm{~cm}$. During this period, nutrient proportions of sprouted barley changed by the growing cycle (Cuddeford, 1989). Sprouting of grains or seeds affects the enzyme activity which leads to hydrolysis proteins, carbohydrates and lipids in their simpler components. The enzyme activity increases total protein and changes the amino acid profile, soluble sugar, crude fiber, certain

\footnotetext{
* Corresponding author: Tel. : +201006031047

E-mail address: megahedmegahed@hotmail.com
}

vitamins and minerals, but decreases starch and total dry mater (Koehler et al., 2007). Furthermore, the advantage of the hydroponic system is to minimize water consumption in fodder production to $2-3 \%$ of that of traditionally field conditions (Al-karaki and Al-Momani, 2011). Productivity measured, on the basis of the input-output balance of barley grain and green fodder yield. The results showed that crude protein $(\mathrm{CP})$, Ash, EE, neutral detergent fiber (NDF), acid detergent fiber (ADF) and water soluble carbohydrate (WSC) were increased whereas organic matter $(\mathrm{OM})$, and non-fiber carbohydrate (NFC) decreased $(p<0.05)$ in the green fodder (GF) when compared with the original grain (Fazaeli et al., 2012). 
Barley seeds which used for producing sprouted green fodder must have a high germination percentage, seed and seedling vigor. To obtain the highest production of sprouted green fodder barley, germination characters must be maximized. Positive and significant correlations were recorded between green fodder yield and seed germination (\%), germination speed index, seedling fresh weight, seedling/ seed ratio, seedling length. Simple linear regression revealed that seed germination (\%), germination index and seedlings fresh weight as significantly contributing variables to variation in green fodder yield (Yousof $\boldsymbol{e t}$ al., 2017). The temperature in growing rooms has been shown to be the most important variable affecting germination (Milbau et al., 2009). Each individual species has a base and ceiling temperature that represents the extremes at which seed germination can occur, below and above these extremes no germination can occur (Finch-Savage and Leubner-Metzger, 2006). The temperature controls germination development of seedlings, and is generally the prime determinant of the duration from sowing to seedling emergence (Flores and Briones, 2001). Khan et al. (2002) found that as temperature increased, the onset of germination was earlier and the time required for $50 \%$ germination decreased. Germination characters of barley enhanced by increasing temperature to $30^{\circ} \mathrm{C}$ (Nejad et al., 2015).

On the other hand, some treatments have recently gained much importance because of their effectiveness and cheapness. It has been found that seed treated with yeast extract (Saccharomyces cerevisiae) improved germination criteria and seedling vigor's (El-Saidy and AbdEl-Hai, 2011; Saker et al., 2015). Seeds treated also with gibberellic acid (GA3) was found to be the most important growth regulator which breaks seed dormancy, promotes germination, internodal length, hypocotyl growth and cell division in cambial zone and increases the size of leaves (Rood $\boldsymbol{e t}$ al., 1990). The objective of this investigation was to determine the effect of seed treatment with yeast extract and gibberellin solution as well as germination temperatures on sprouted green fodder production.

\section{MATERIALS AND METHODS}

Laboratory experiments were carried out at Seed Technology Research Department, Field Crop Research Institute ,Agricultural Research Center, Egypt, in 2017 season, in order to determine the effect of seed soaking in yeast extract (1,2 and $3 \mathrm{~g} / \mathrm{l})$ and gibberellin solution (4, 8 and $12 \mathrm{ppm})$, as well as distilled water at various temperatures of germination rooms $\left(15,20,25,30\right.$ and $\left.35^{\circ} \mathrm{C}\right)$ on seed germination (\%), seedling characters, sprouted green fodder yield $\left(\mathrm{Kg} / \mathrm{m}^{2}\right)$ and chemical characteristics of barley green fodder. Barley seed (CV Giza 136) were obtained from Barley Research Department, Field Crops Research Institute.

\section{Preparation of Yeast Extract and Gibberellin Solution}

Active dry yeast was dissolved in water at rates 1,2 and $3 \mathrm{~g} / \mathrm{l}$ followed by adding sugar at ratio $1: 1$. This ratio is suitable to get the highest production rate of yeast (each $\mathrm{ml}$ yeast contained about 12000 of yeast cells) and kept overnight for activation and reproduction of yeast. Yeast extract was prepared by using a technique allowed yeast cells (pure dry yeast) to be grown and multiplied efficiently during conducive aerobic and nutritional conditions that allowed to produce beneficial bio constituent, (carbohydrates, sugars, proteins, amino acids, fatty acids, hormones, etc.), then these constituents could release out of yeast cells in ready form by two cycles of freezing and thawing for disruption of yeast cells and releasing their content. Such technique for yeast preparation modified after (Spencer $\boldsymbol{e t} \boldsymbol{a l}$, 1983). Gibberellin solution was prepared by dissolving (4, 8 and $12 \mathrm{mg}$ ) gibberellins powder each in 1 liter of distilled water to get gibberellin solution (4, 8 and $12 \mathrm{ppm})$.

\section{Seed Treatment and Measurements}

Barley seeds (Hordium vulgare L.) of Giza 136 cultivar were soaked in gibberellin solution (4, 8 and $12 \mathrm{ppm})$, yeast extracts $(1,2$ and $3 \mathrm{~g} / \mathrm{l})$ and distilled water for $1 \mathrm{hr}$. Germination tests were carried out for seed soaking treatments and dry seed in sterilized Petri dishes $(150 \times 15 \mathrm{~mm})$ covered at the bottom with two sheets of Whatman No.1 filter paper that had been 
autoclaved. Each dish included 50 seeds incubated in growth chamber at 15, 20, 25, 30 and $35^{\circ} \mathrm{C}$ and germination tests were performed according to Intentional Seed Testing Association (ISTA, 2004). Measurements were made on seed germination (\%), germinated abnormally (\%), non germinated seeds $(\%)$, germination speed index (GSI), seedling length $(\mathrm{cm})$, seedlings fresh weight $(\mathrm{g})$, seedlings dry weight $(\mathrm{g})$ and seedling/seed weight ratio. The coefficient of germination uniformity (CGU) is given by $\Sigma \mathrm{N} / \Sigma\left((\mathrm{MTG}-\mathrm{T})^{2} \cdot \mathrm{N}\right)$. MTG is equal to $\Sigma(\mathrm{T} \cdot \mathrm{N}) /$ $\Sigma \mathrm{N}$, where $\mathrm{T}$ is the time in days, starting from day 0 , the beginning of the test, and $\mathrm{N}$ is the number of seeds completing germination on day $\mathrm{t}$ (Bewley and Black, 1994).

\section{Green Fodder Yield Preparation}

Steel trays with dimensions $(30 \mathrm{~cm} \times 40 \mathrm{~cm}$ $\times 4 \mathrm{~cm}$ ) were used for growing barley seeds to produce green fodder. Seeds of Giza 136 barley $\mathrm{cv}$ were cleaned from dust and any inert materials, then sterilized by sodium hypochloride $5 \%$, for 5 minutes, then it washed well in tap water before soaking overnight before planting. The seeding rate was about $480 \mathrm{~g} /$ tray (equivalent to about $4.0 \mathrm{Kg} / \mathrm{m}^{2}$ ) according to Al-Hashmi (2008). Seeds were spread on trays, which were stacked on shelves of the germinator. The temperature inside the germinator were $(15,20$, 25,30 and $35^{\circ} \mathrm{C}$ ) and the relative humidity adjusted about $70 \%$. The trays were irrigated twice a day. Green fodder yield $\left(\mathrm{Kg} / \mathrm{m}^{2}\right)$ and yield/seed ratio were estimated after 7 days.

\section{Chemical Parameters}

Dry matter $\left(\mathrm{Kg} / \mathrm{m}^{2}\right)$ was determined by drying the samples at $60^{\circ} \mathrm{C}$ in a forced-air oven for 48 hr., dry matter $(\%)$ was calculated by dividing dry matter weight on green fodder yield weight multiple by 100 . Crude protein (\%), ether extract $(\%)$, and ash $(\%)$ were measured according to the Association of Official Agriculture Chemists (AOAC, 1980). Organic matter (\%) was calculated as [100-Ash (\%)]. The non-fiber carbohydrate (NFC) was estimated as: [100- (CP+Ash+ EE + NDF)]. Neutral detergent fiber (NDF) and acid detergent fiber (ADF) were determined according to Van Soest et al. (1991).

All data were subjected to the statistical analysis according to the technique of analysis of variance (ANOVA) of completely randomized design, as described by Gomez and Gomez (1984). The data of green fodder yield $\left(\mathrm{Kg} / \mathrm{m}^{2}\right)$, germination (\%), germination speed index (GSI), seedling length $(\mathrm{cm})$ and seedlings fresh weight $(\mathrm{g})$ were also subjected to procedures of simple correlation, partial regression and stepwise regression techniques outlined by (Draper and Smith, 1966)

The partial regression equation was:

$$
\mathrm{Y}=\mathrm{a}+\mathrm{b} \mathrm{X}
$$

Where:

$\mathrm{Y}=$ Green fodder yield

$\mathrm{a}=$ intercept constant

$\mathrm{b}=$ regression coefficient of $\mathrm{X}$

$\mathrm{X}=$ the independent variable such as standard seed germination, germination speed index .....ect.

The multiple regression equation was:

$$
Y=a+b_{1} X_{1}+b_{2} X_{2}+b_{3} X_{3}+\ldots \ldots \ldots b_{n} X_{n}
$$

Where:

$\mathrm{n}$ is the number of independent variables

\section{RESULTS AND DISCUSSION}

The results in Table 1 show seed germination $(\%)$, abnormal germinated seeds (\%), non germinated seeds (\%), coefficient of germination uniformity (CGU) and germination speed index (GSI) of barley cv Giza 136 as affected by seed soaking treatments and germination temperature. Barley seed soaking in gibberellin solution (12 ppm) was superior compared with other seed treatments and seed soaking in yeast extract $(3 \mathrm{~g} / \mathrm{l})$ came in the second rank without significant differences between gibberellin and yeast extract treatments. No significant differences were obtained among seed soaking treatments as to abnormal germinated seeds (\%) and coefficient of germination uniformity (CGU). On the other hand, untreated seed recorded the lowest parameters. With respect to the effect of germination temperature, the highest parameters concerning germination characters were obtained by germinating seeds at $35^{\circ} \mathrm{C}$ followed by those at $30^{\circ} \mathrm{C}$ without significant differences 
Table 1. Seed germination $(\%)$, abnormal germinated seed $(\%)$, non germinated seed $(\%)$, coefficient of germination uniformity (CGU) and germination speed index (GSI) of barley as affected by seed soaking treatment and germination temperature

\begin{tabular}{|c|c|c|c|c|c|}
\hline \multirow[t]{2}{*}{ Treatment } & \multicolumn{5}{|c|}{ Germination characters } \\
\hline & $\begin{array}{c}\text { Seed } \\
\text { germination } \\
(\%)\end{array}$ & $\begin{array}{c}\text { Abnormal } \\
\text { germinated } \\
\text { seed }(\%)\end{array}$ & $\begin{array}{c}\text { Non } \\
\text { germinated } \\
\text { seed }(\%)\end{array}$ & CGU & GSI \\
\hline \multicolumn{6}{|l|}{ Seed soaking } \\
\hline Untreated seed & 74 & 8 & 18 & 2.31 & 56.0 \\
\hline Distilled water & 75 & 8 & 17 & 2.37 & 57.3 \\
\hline Gibberellin solution (4 ppm) & 76 & 7 & 17 & 2.41 & 57.5 \\
\hline Gibberellin solution (8 ppm) & 77 & 6 & 17 & 2.48 & 59.1 \\
\hline Gibberellin solution (12 ppm) & 81 & 6 & 13 & 2.61 & 61.4 \\
\hline Yeast extract $(1 \mathrm{~g} / \mathrm{l})$ & 75 & 7 & 18 & 2.38 & 57.4 \\
\hline Yeast extract ( $2 \mathrm{~g} / \mathrm{l})$ & 77 & 7 & 16 & 2.43 & 58.8 \\
\hline Yeast extract ( $3 \mathrm{~g} / \mathrm{l})$ & 79 & 6 & 15 & 2.59 & 60.9 \\
\hline F test & $* *$ & NS & $*$ & NS & $*$ \\
\hline LSD 5\% & 3 & - & 2 & - & 1.4 \\
\hline \multicolumn{6}{|l|}{ Germination temperature } \\
\hline $15^{\circ} \mathrm{C}$ & 70 & 10 & 20 & 2.03 & 53.1 \\
\hline $20^{\circ} \mathrm{C}$ & 74 & 7 & 19 & 2.42 & 57.9 \\
\hline $25^{\circ} \mathrm{C}$ & 77 & 7 & 16 & 2.53 & 60.1 \\
\hline $30^{\circ} \mathrm{C}$ & 81 & 5 & 14 & 2.59 & 60.6 \\
\hline $35^{\circ} \mathrm{C}$ & 82 & 5 & 13 & 2.64 & 61.1 \\
\hline F-test & $* *$ & $* *$ & $*$ & $*$ & $*$ \\
\hline LSD $5 \%$ & 3 & 3 & 3 & 0.21 & 1.9 \\
\hline
\end{tabular}

$\mathrm{NS}, *$ and $* *$ are not significant, significant at 5 and $1 \%$, respectively.

between them. On contrast, the lowest parameters were obtained at $15^{\circ} \mathrm{C}$. The results in Tables 2 and 3 show the effect of seed soaking and germination temperature on seedling characters namely seedling length $(\mathrm{cm})$, seedling fresh weight $(\mathrm{g})$, seedling dry weight $(\mathrm{g})$, seedling/seed ratio, green fodder yield $\left(\mathrm{Kg} / \mathrm{m}^{2}\right)$, yield/seed ratio, dry matter yield $\left(\mathrm{Kg} / \mathrm{m}^{2}\right)$, dry matter $(\%)$, ash $(\%)$, organic matter $(\%)$, crude protein $(\%)$, neutral detergent fiber (NDF) $(\%)$, acid detergent fiber (\%) (ADF), ether extract (\%) (EE) and non fiber carbohydrate (\%) (NCF). Seed soaking in gibberellin solution (12 ppm) and yeast extract $(3 \mathrm{~g} / \mathrm{l})$ exceeded other treatments without significant differences between them. Insignificant differences were obtained between seed soaking in distilled water and soaking in gibberellin solution (4 ppm). On the other hand, untreated seed gave the lowest values of each of seedling length $(\mathrm{cm})$, seedling fresh weight $(\mathrm{g})$, seedling dry weight $(\mathrm{g})$, seedling/seed ratio, green fodder yield $\left(\mathrm{Kg} / \mathrm{m}^{2}\right)$, yield/seed ratio, dry matter $\left(\mathrm{Kg} / \mathrm{m}^{2}\right)$, dry matter $(\%)$, neutral detergent fiber (NDF) (\%), ether extract (\%) and highest non-fiber carbohydrate (\%) (NCF). Germination temperature at $35^{\circ} \mathrm{C}$ significantly exceeded other temperature in all parameters of Tables 2 and 3 and it was followed by seed germination at $30^{\circ} \mathrm{C}$ without significant differences between them, whereas all parameters recorded at previous temperatures were high and significant compared with those recorded at lower temperatures $\left(15,20,25^{\circ} \mathrm{C}\right)$. Furthermore, insignificant differences were recorded among germination temperature for ash $(\%)$, organic matter $(\%)$, crude protein $(\%)$, and 
Table 2. Seedling length $(\mathrm{cm})$, seedling fresh weight $(\mathrm{g})$, seedling dry weight $(\mathrm{g})$, seedling /seed ratio, green fodder yield $\left(\mathrm{Kg} / \mathrm{m}^{2}\right)$, yield/seed ratio and dry matter yield $\left(\mathrm{Kg} / \mathrm{m}^{2}\right)$ of barley as affected by seed soaking treatment and germination temperature

\begin{tabular}{|c|c|c|c|c|c|c|c|}
\hline \multirow[t]{2}{*}{ Treatment } & \multicolumn{4}{|c|}{ Seedling characters } & \multicolumn{3}{|c|}{ Green fodder characters } \\
\hline & 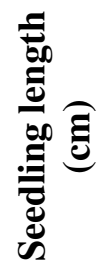 & 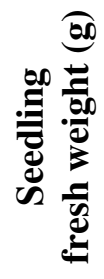 & 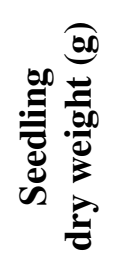 & 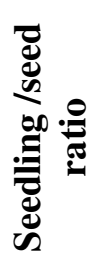 & 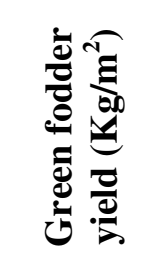 & 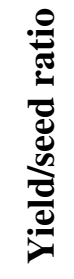 & 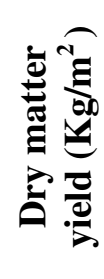 \\
\hline \multicolumn{8}{|l|}{ Seed soaking } \\
\hline Untreated seed & 15.0 & 4.77 & 0.691 & 10.9 & 22.493 & 5.4 & 2.978 \\
\hline Distilled water & 15.6 & 5.06 & 0.739 & 11.3 & 22.736 & 5.8 & 3.067 \\
\hline Gibberellin solution (4 ppm) & 15.7 & 5.23 & 0.748 & 11.6 & 23.184 & 5.9 & 3.162 \\
\hline Gibberellin solution (8 ppm) & 16.2 & 5.47 & 0.770 & 12.3 & 24.768 & 6.2 & 3.462 \\
\hline Gibberellin solution (12 ppm) & 17.1 & 5.64 & 0.830 & 12.8 & 25.862 & 6.5 & 3.804 \\
\hline Yeast extract $(1 \mathrm{~g} / \mathrm{l})$ & 15.7 & 5.06 & 0.730 & 11.5 & 23.245 & 5.8 & 3.156 \\
\hline Yeast extract $(2 \mathrm{~g} / \mathrm{l})$ & 16.0 & 5.31 & 0.763 & 12.0 & 24.126 & 6.0 & 3.363 \\
\hline Yeast extract $(3 g / l)$ & 16.8 & 5.62 & 0.828 & 12.5 & 25.512 & 6.4 & 3.727 \\
\hline F-test & $* *$ & $* *$ & $*$ & $* *$ & $* *$ & $*$ & $*$ \\
\hline LSD 5\% & 0.7 & 0.42 & 0.051 & 0.6 & 0.682 & 0.4 & 0.175 \\
\hline \multicolumn{8}{|l|}{ Germination temperature } \\
\hline $15^{\circ} \mathrm{C}$ & 14.3 & 4.45 & 0.668 & 10.5 & 22.212 & 5.2 & 2.794 \\
\hline $20^{\circ} \mathrm{C}$ & 15.6 & 5.03 & 0.744 & 11.6 & 23.097 & 5.8 & 3.116 \\
\hline $25^{\circ} \mathrm{C}$ & 16.4 & 5.49 & 0.773 & 11.9 & 23.689 & 6.2 & 3.376 \\
\hline $30^{\circ} \mathrm{C}$ & 16.7 & 5.58 & 0.789 & 12.4 & 25.211 & 6.3 & 3.658 \\
\hline $35^{\circ} \mathrm{C}$ & 17.1 & 5.81 & 0.831 & 13.0 & 25.744 & 6.5 & 3.771 \\
\hline F-test & $* *$ & $* *$ & $* *$ & $* *$ & $* *$ & $*$ & $* *$ \\
\hline LSD 5\% & 0.9 & 0.52 & 0.073 & 0.8 & 0.754 & 0.6 & 0.265 \\
\hline
\end{tabular}

$\mathrm{NS}, *$ and $* *$ are not significant, significant at 5 and $1 \%$, respectively. 
Table 3. Dry matter $(\%)$, ash $(\%)$, organic matter $(\%)$, crude protein $(\%)$, neutral detergent fiber (\%) (NDF), acid detergent fiber (\%) (ADF), ether extract (\%) ment and germination temperature

\begin{tabular}{|c|c|c|c|c|c|c|c|c|}
\hline \multirow[t]{2}{*}{ Treatment } & \multicolumn{8}{|c|}{ Chemical characters } \\
\hline & $\begin{array}{c}\text { Dry } \\
\text { matter } \\
(\%)\end{array}$ & $\begin{array}{l}\text { Ash } \\
(\%)\end{array}$ & $\begin{array}{c}\text { Organic } \\
\text { matter } \\
(\%)\end{array}$ & $\begin{array}{c}\text { Crude } \\
\text { protein } \\
(\%)\end{array}$ & $\begin{array}{l}\text { NDF } \\
(\%)\end{array}$ & $\begin{array}{c}\text { ADF } \\
(\%)\end{array}$ & $\begin{array}{l}\text { EE } \\
(\%)\end{array}$ & $\begin{array}{l}\text { NCF } \\
(\%)\end{array}$ \\
\hline \multicolumn{9}{|l|}{ Seed soaking } \\
\hline Untreated seed & 13.24 & 3.21 & 96.79 & 13.43 & 29.54 & 14.58 & 2.13 & 51.78 \\
\hline Distilled water & 13.49 & 3.42 & 96.58 & 13.44 & 29.83 & 14.72 & 2.38 & 51.12 \\
\hline Gibberellin solution (4 ppm) & 13.64 & 3.58 & 96.42 & 13.46 & 30.28 & 14.75 & 2.44 & 50.14 \\
\hline Gibberellin solution (8 ppm) & 13.98 & 3.76 & 96.24 & 13.50 & 31.00 & 14.87 & 2.63 & 48.93 \\
\hline Gibberellin solution (12 ppm) & 14.71 & 3.78 & 96.22 & 13.52 & 32.25 & 14.96 & 2.74 & 47.71 \\
\hline Yeast extract $(1 \mathrm{~g} / \mathrm{l})$ & 13.58 & 3.49 & 96.51 & 13.42 & 30.15 & 14.75 & 2.40 & 50.54 \\
\hline Yeast extract ( $2 \mathrm{~g} / \mathrm{l})$ & 13.94 & 3.72 & 96.28 & 13.48 & 31.04 & 14.84 & 2.58 & 49.18 \\
\hline Yeast extract $(3 \mathrm{~g} / \mathrm{l})$ & 14.61 & 3.75 & 96.25 & 13.53 & 31.94 & 14.93 & 2.69 & 48.09 \\
\hline F test & $* *$ & NS & NS & NS & $* *$ & NS & $*$ & $* *$ \\
\hline LSD 5\% & 0.37 & - & - & - & 0.65 & - & 0.29 & 0.52 \\
\hline \multicolumn{9}{|l|}{ Germination temperature } \\
\hline $15^{\circ} \mathrm{C}$ & 12.58 & 3.20 & 96.80 & 13.42 & 28.37 & 14.71 & 2.24 & 52.77 \\
\hline $20^{\circ} \mathrm{C}$ & 13.49 & 3.46 & 96.54 & 13.44 & 29.37 & 14.79 & 2.42 & 51.31 \\
\hline $25^{\circ} \mathrm{C}$ & 14.25 & 3.71 & 96.29 & 13.50 & 31.19 & 14.80 & 2.51 & 49.09 \\
\hline $30^{\circ} \mathrm{C}$ & 14.51 & 3.74 & 96.26 & 13.50 & 31.86 & 14.82 & 2.59 & 48.31 \\
\hline $35^{\circ} \mathrm{C}$ & 14.65 & 3.81 & 96.19 & 13.51 & 32.97 & 14.92 & 2.71 & 47.00 \\
\hline F-test & $* *$ & NS & NS & NS & $* *$ & NS & NS & $* *$ \\
\hline LSD 5\% & 0.66 & - & - & - & 0.81 & - & - & 0.61 \\
\hline
\end{tabular}

$\mathrm{NS}, *$ and $* *$ are not significant, significant at 5 and $1 \%$, respectively.

acid detergent fiber (\%) (ADF) and ether extract (\%) (EE). Germination at $15^{\circ} \mathrm{C}$ recorded the lowest values characters in Tables 2 and 3.

Tables 4,5 and 6 show the interaction effect between seed soaking treatment and germination temperature on seed germination (\%), germination speed index and seedling length $(\mathrm{cm})$. Soaking barley seeds in gibberellin solution $(12 \mathrm{ppm})$ and germination at $35^{\circ} \mathrm{C}$ temperature exceeded other treatments. No. significant differences in seed germination (\%) were obtained between untreated seed, soaking in distilled and soaking treatment at all temperatures under study. However, insignificant differences were obtained between soaking in gibberellin solution (12 ppm) treatment and soaking in yeast extract $(3 \mathrm{~g} / \mathrm{l})$ at $35^{\circ} \mathrm{C}$. While, untreated seed and germination at $15^{\circ} \mathrm{C}$ produced the lowest values of previous parameters.

Tables 7, 8 and 9 show that seed soaking in gibberellin solution (12 ppm) and yeast extract (3 $\mathrm{g} / \mathrm{l})$ with germination at $35^{\circ} \mathrm{C}$ recorded the heaviest seedling fresh weight $(\mathrm{g})$, highest values of seedling/seed ratio and green fodder yield $\left(\mathrm{Kg} / \mathrm{m}^{2}\right)$ parameters without significant differences between them. On the other hand, the lightest seedling fresh weight $(\mathrm{g})$ and the lowest seedling/seed ratio as well as green fodder yield $\left(\mathrm{Kg} / \mathrm{m}^{2}\right)$ parameters were recorded by germinating untreated seed at $15^{\circ} \mathrm{C}$ and soaking in distilled water without significant differences between them. 
Zagazig J. Agric. Res., Vol. 45 No. (4) 2018

Table 4. Seed germination (\%) of barley as affected by the interaction between seed soaking treatments and germination temperature

\begin{tabular}{lccccc}
\hline Seed soaking & \multicolumn{5}{c}{ Germination temperatures } \\
\cline { 2 - 6 } & $\mathbf{1 5}^{\mathbf{0}} \mathbf{C}$ & $\mathbf{2 0}^{\mathbf{0}} \mathbf{C}$ & $\mathbf{2 5}^{\mathbf{0}} \mathbf{C}$ & $\mathbf{3 0}^{\mathbf{0}} \mathbf{C}$ & $\mathbf{3 5}^{\mathbf{0}} \mathbf{C}$ \\
\hline Untreated seed & 67 & 71 & 75 & 77 & 78 \\
Distilled water & 69 & 73 & 76 & 79 & 80 \\
Gibberellin solution (4 ppm) & 69 & 74 & 76 & 81 & 82 \\
Gibberellin solution (8 ppm) & 70 & 74 & 77 & 81 & 83 \\
Gibberellin solution (12 ppm) & 73 & 76 & 80 & 86 & 89 \\
Yeast extract (1 g/l) & 69 & 73 & 76 & 79 & 80 \\
Yeast extract (2 g/l) & 70 & 73 & 77 & 80 & 82 \\
Yeast extract $(\mathbf{3}$ g/l) & 71 & 75 & 78 & 83 & 86 \\
LSD at 5\% & & & 4 & & \\
\hline
\end{tabular}

Table 5. Germination speed index (GSI) of barley as affected by the interaction between seed soaking treatments and germination temperatures

\begin{tabular}{|c|c|c|c|c|c|}
\hline \multirow[t]{2}{*}{ Seed soaking } & \multicolumn{5}{|c|}{ Germination temperatures } \\
\hline & $15^{\circ} \mathrm{C}$ & $20^{\circ} \mathrm{C}$ & $25^{\circ} \mathrm{C}$ & $3^{\circ} \mathrm{C}$ & $35^{\circ} \mathrm{C}$ \\
\hline Untreated seed & 49.6 & 55.3 & 57.8 & 58.4 & 58.9 \\
\hline Distilled water & 51.6 & 56.9 & 59.0 & 59.4 & 59.8 \\
\hline Gibberellin solution (4 ppm) & 51.9 & 57.0 & 59.2 & 59.5 & 59.8 \\
\hline Gibberellin solution (8 ppm) & 54.7 & 58.5 & 60.3 & 60.8 & 61.1 \\
\hline Gibberellin solution (12 ppm) & 55.6 & 60.5 & 63.1 & 63.4 & 64.4 \\
\hline Yeast extract (1 g/l) & 51.8 & 56.9 & 59.2 & 59.5 & 59.7 \\
\hline Yeast extract ( $2 \mathrm{~g} / \mathrm{l})$ & 54.5 & 57.9 & 60.0 & 60.6 & 61.0 \\
\hline Yeast extract $(3 \mathrm{~g} / \mathrm{l})$ & 55.0 & 60.0 & 62.4 & 63.0 & 64.0 \\
\hline LSD at $5 \%$ & \multicolumn{5}{|c|}{1.5} \\
\hline
\end{tabular}


Table 6. Seedling length $(\mathrm{cm})$ of barley as affected by the interaction between seed soaking treatments and germination temperatures

\begin{tabular}{lccccc}
\hline Seed soaking & \multicolumn{5}{c}{ Germination temperatures } \\
\cline { 2 - 6 } & $\mathbf{1 5}^{\mathbf{0}} \mathbf{C}$ & $\mathbf{2 0}^{\mathbf{0}} \mathbf{C}$ & $\mathbf{2 5}^{\mathbf{0}} \mathbf{C}$ & $\mathbf{3 0}^{\mathbf{0}} \mathbf{C}$ & $\mathbf{3 5}^{\mathbf{0}} \mathbf{C}$ \\
\hline Untreated seed & 13.2 & 14.7 & 15.5 & 15.8 & 16.0 \\
Distilled water & 13.9 & 15.3 & 16.0 & 16.3 & 16.4 \\
Gibberellin solution (4 ppm) & 14.0 & 15.4 & 16.1 & 16.3 & 16.7 \\
Gibberellin solution (8 ppm) & 14.6 & 15.8 & 16.4 & 16.9 & 17.4 \\
Gibberellin solution (12 ppm) & 15.3 & 16.5 & 17.4 & 18.0 & 18.4 \\
Yeast extract (1 g/l) & 13.9 & 15.4 & 16.3 & 16.2 & 16.5 \\
Yeast extract (2 g/l) & 14.4 & 15.6 & 16.5 & 16.6 & 17.0 \\
Yeast extract $\mathbf{( 3}$ g/l) & 14.9 & 16.3 & 17.1 & 17.6 & 18.1 \\
LSD at 5\% & & & 1.2 & & \\
\hline
\end{tabular}

Table 7. Seedling fresh weight (g) of barley as affected by the interaction between seed soaking treatments and germination temperature

\begin{tabular}{lccccc}
\hline Seed soaking & \multicolumn{5}{c}{ Germination temperature } \\
\cline { 2 - 6 } & $\mathbf{1 5}^{\mathbf{0}} \mathbf{C}$ & $\mathbf{2 0}^{\mathbf{0}} \mathbf{C}$ & $\mathbf{2 5}^{\mathbf{0}} \mathbf{C}$ & $\mathbf{3 0}^{\mathbf{0}} \mathbf{C}$ & $\mathbf{3 5}^{\mathbf{0}} \mathbf{C}$ \\
\hline Untreated seed & 4.12 & 4.54 & 4.95 & 5.08 & 5.17 \\
Distilled water & 4.25 & 4.87 & 5.26 & 5.37 & 5.54 \\
Gibberellin solution (4 ppm) & 4.41 & 4.96 & 5.47 & 5.55 & 5.78 \\
Gibberellin solution (8 ppm) & 4.59 & 5.18 & 5.62 & 5.76 & 6.21 \\
Gibberellin solution (12 ppm) & 4.75 & 5.46 & 5.84 & 5.91 & 6.26 \\
Yeast extract (1 g/l) & 4.37 & 4.91 & 5.22 & 5.35 & 5.46 \\
Yeast extract (2 g/l) & 4.47 & 5.09 & 5.57 & 5.63 & 5.78 \\
Yeast extract $(\mathbf{3}$ g/l) & 4.63 & 5.28 & 5.95 & 5.99 & 6.25 \\
LSD at 5\% & & & 0.54 & & \\
\hline
\end{tabular}


Table 8. Seedling/seed ratio of barley as affected by the interaction between seed soaking treatments and germination temperature

\begin{tabular}{lccccc}
\hline Seed soaking & \multicolumn{5}{c}{ Germination temperatures } \\
\cline { 2 - 6 } & $\mathbf{1 5}^{\mathbf{0}} \mathbf{C}$ & $\mathbf{2 0}^{\mathbf{0}} \mathbf{C}$ & $\mathbf{2 5}^{\mathbf{0}} \mathbf{C}$ & $\mathbf{3 0}^{\mathbf{0}} \mathbf{C}$ & $\mathbf{3 5}^{\mathbf{0}} \mathbf{C}$ \\
\hline Untreated seed & 9.7 & 10.7 & 11.1 & 11.3 & 11.8 \\
Distilled water & 10.2 & 11.3 & 11.5 & 11.5 & 12.1 \\
Gibberellin solution (4 ppm) & 10.5 & 11.3 & 11.7 & 12.0 & 12.7 \\
Gibberellin solution (8 ppm) & 10.7 & 11.9 & 12.3 & 12.8 & 13.6 \\
Gibberellin solution (12 ppm) & 11.3 & 12.2 & 12.9 & 13.5 & 13.9 \\
Yeast extract (1 g/l) & 10.2 & 11.3 & 11.5 & 11.9 & 12.5 \\
Yeast extract (2 g/l) & 10.4 & 11.8 & 12 & 12.5 & 13.5 \\
Yeast extract $\mathbf{( 3}$ g/l) & 11.1 & 12.1 & 12.5 & 13.3 & 13.7 \\
LSD at 5\% & & & 1.0 & & \\
\hline
\end{tabular}

Table 9. Green fodder yield $\left(\mathrm{Kg} / \mathrm{m}^{2}\right)$ of barley as affected by the interaction between seed soaking treatments and germination temperature

\begin{tabular}{lccccc}
\hline Seed soaking & \multicolumn{5}{c}{ Germination temperatures } \\
\cline { 2 - 6 } & $\mathbf{1 5}^{\mathbf{0}} \mathbf{C}$ & $\mathbf{2 0}^{\mathbf{0}} \mathbf{C}$ & $\mathbf{2 5}^{\mathbf{0}} \mathbf{C}$ & $\mathbf{3 0}^{\mathbf{0}} \mathbf{C}$ & $\mathbf{3 5}^{\mathbf{0}} \mathbf{C}$ \\
\hline Untreated seed & 20.935 & 21.285 & 22.274 & 23.544 & 24.425 \\
Distilled water & 21.085 & 21.946 & 22.348 & 23.776 & 24.528 \\
Gibberellin solution (4 ppm) & 21.485 & 22.301 & 22.756 & 24.362 & 25.018 \\
Gibberellin solution (8 ppm) & 22.857 & 23.914 & 24.358 & 25.849 & 26.862 \\
Gibberellin solution (12 ppm) & 23.774 & 24.821 & 25.772 & 27.042 & 27.902 \\
Yeast extract (1 g/l) & 21.663 & 22.564 & 22.872 & 23.876 & 25.254 \\
Yeast extract (2 g/l) & 22.250 & 23.202 & 23.742 & 25.22 & 26.216 \\
Yeast extract (3 g/l) & 23.652 & 24.742 & 25.391 & 26.421 & 27.354 \\
LSD at 5\% & & & 0.976 & & \\
\hline
\end{tabular}


Table 10 show the simple correlation coefficient for seed germination (\%), germination speed index, seedling length and seedling fresh weight towards green fodder yield. Positive and significant correlation coefficients were recorded between green fodder yield and each of seed germination (\%), germination speed index, seedling length and seedling fresh weight which were 0.939, 0.879, 0.892 and 0.928 , respectively.

The results in Tables 11 and 12 show the stepwise regression analysis of seed germination $(\%)$, germination speed index, and seedling length and seedling fresh weight towards green fodder yield. The prediction equation for green fodder yield was computed as follow:

$$
\mathrm{Y}=3.957+0.129 \mathrm{X} 1+0.251 \mathrm{X} 2+1.263 \mathrm{X} 3
$$

Where :

$\mathrm{Y}=$ Green fodder yield

$3.957=$ Constant.

$\mathrm{X} 1=$ Seed germination $(\%)$

$\mathrm{X} 2$ = Seedling length .

X3 = Seedling fresh weight.

The relative contribution for seed germination (\%), germination speed index, seedling length $(\mathrm{cm})$ and seedling fresh weight towards green fodder yield was $83.7 \%$, simple correlation of all previous characters together with green fodder yield $\left(\mathrm{Kg} / \mathrm{m}^{2}\right)$ was 0.901 (Table 11). seed germination (\%), seedling length and seedling fresh weight were significantly contributing characters as to variation in green fodder yields, seed germination (\%) was the first character $\left(\mathrm{R}^{2}=82.6 \%\right)$ followed by seedling fresh weight $\left(\mathrm{R}^{2}=82.4 \%\right)$ and seedling length $\left(\mathrm{R}^{2}=69.1 \%\right)$. While, germination speed index was insignificantly contributing to green fodder yield and recorded the lowest ( $\mathrm{R}$ squared which was $2.87 \%$ (Table 12).
In the present study, increasing temperature from $15^{\circ} \mathrm{C}$ to $35^{\circ} \mathrm{C}$ had a positive effect on germination parameters and final sprouted green fodder yield of barley. Temperature affects seed germination in three primary ways: seed moisture, hormone production, and enzyme activity, chemical signaling regulates production of enzymes, which is in turn regulated by temperature (Finch-Savage and LeubnerMetzger, 2006). The temperature has an impact on a number of processes that regulate seed germination, including membrane permeability and the activity of membrane-bound and cytosolic enzymes (Tlig et al., 2008). Germination rate usually increases linearly with increasing temperature up to an optimum point, and then decreases linearly to a ceiling temperature (Bradford, 2002).

The results indicated that soaking barley seeds in gibberellin solution (12 ppm) gave the highest values of parameters under all temperatures $\left(15,20,25, \quad 30\right.$ and $\left.35^{\circ} \mathrm{C}\right)$ compared to other seed treatments. GA stimulates hydrolytic enzymes that are needed for the degradation of the cells surrounding the radicle and thus speeds germination by promoting seedling elongation growth of cereal seeds (Rood et al., 1990). Yeast extract came in the second rank after gibberellin solution and proved their efficiency to record satisfactory production of barley sprouted green fodder. Many researchers provided explanations for yeast extract mode of action, El-Tohamy and El-Greadly (2007) showed that yeast extract contained a higher contents of cytokinins, IAA and GA3. It was reported about its stimulatory effects on cell division and enlargement, protein and nucleic acid synthesis and vitamins (Castelfranco and Beale, 1983). It could be concluded that seed soaking in gibberellin solution (12 ppm) and yeast extract $(3 \mathrm{~g} / \mathrm{l})$ is a good seed treatment for different temperatures to maximize sprouted green fodder yield of barley.

Table 10. Simple correlation coefficient $(\mathbf{R})$ for seed germination $(\%)$, germination speed index, seedling length and seedling fresh weight towards green fodder yield

\begin{tabular}{lccrr}
\hline $\mathbf{X}$ & $\begin{array}{c}\text { Seed germination Germination speed } \\
\text { index }\end{array}$ & $\begin{array}{c}\text { Seedling } \\
\text { length }(\mathbf{c m})\end{array}$ & $\begin{array}{c}\text { Seedling fresh } \\
\text { weight }(\mathbf{g})\end{array}$ \\
\hline Green fodder yield $\left(\mathbf{K g} / \mathbf{m}^{2}\right)$ & $0.939 * *$ & $0.879 * *$ & $0.892 * *$ & $0.928 * *$ \\
\hline
\end{tabular}


Table 11. $R, R$ square and adjusted $R$ square of contribution germination (\%), germination speed index, seedling length $(\mathrm{cm})$ and seedling fresh weight $(\mathrm{g})$ towards green fodder yield $\left(\mathrm{Kg} / \mathrm{m}^{2}\right)$ according to stepwise regression analysis

\begin{tabular}{lccc}
\hline $\mathbf{R}$ & R square & Adjusted R square & Std. Error of the estimate \\
\hline 0.901 & 0.854 & 0.837 & 0.736 \\
\hline
\end{tabular}

Table 12. Effective and inefficient characters affected green fodder yield according to stepwise regression analysis

\begin{tabular}{|c|c|c|c|c|c|c|c|c|}
\hline \multirow[t]{2}{*}{ Accepted variables } & \multicolumn{2}{|c|}{$\begin{array}{c}\text { Unstandardized Standardized } \\
\text { coefficients coefficients }\end{array}$} & \multirow[t]{2}{*}{$\mathbf{T}$} & \multirow[t]{2}{*}{ Sig. } & \multirow[t]{2}{*}{$\begin{array}{l}\text { Effective } \\
\text { character }\end{array}$} & \multirow{2}{*}{$\begin{array}{c}\text { Partial } \\
\text { regression } \\
\left(\mathbf{R}^{2}\right)\end{array}$} & \multirow{2}{*}{$\begin{array}{l}\text { Inefficient } \\
\text { character }\end{array}$} & \multirow{2}{*}{$\begin{array}{c}\text { Partial } \\
\text { regression } \\
\left(\mathbf{R}^{2}\right)\end{array}$} \\
\hline & B Std. Error & B Std. Error & & & & & & \\
\hline Constant & 3.957 & 8.75 & 0.994 & 0.327 & Seed & & \multirow{5}{*}{$\begin{array}{l}\text { Germination } \\
\text { speed index } \\
\text { (GSI) }\end{array}$} & \multirow{5}{*}{2.87} \\
\hline Seed germination $(\%)$ & 0.129 & 0.24 & 4.653 & 0.003 & \multirow{3}{*}{$\begin{array}{l}\text { Germination } \\
\qquad(\%) \\
\text { Seedling } \\
\text { length }\end{array}$} & 82.6 & & \\
\hline Germination speed index & 0.008 & 0.471 & 0.067 & 0.947 & & & & \\
\hline Seedling length & 0.251 & 3.03 & 4.335 & 0.027 & & 69.1 & & \\
\hline Seedling fresh weight & 1.263 & 3.33 & 4.011 & 0.042 & $\begin{array}{l}\text { Seedling fresh } \\
\text { weight }\end{array}$ & 82.4 & & \\
\hline
\end{tabular}

\section{REFERENCES}

Al-Hashmi, M. M. (2008). Hydroponic green fodder production in the Arabian Gulf Region. M.Sc. Thesis, Fac. Graduate Studies, Arabian Gulf Univ.

Al-Karaki, G. N. and N. Al-Momani (2011). Evaluation of some barley $\mathrm{cv}$ for green fodder production and water use efficiency under hydroponic conditions. Jordan J. Agric. Sci., 7 (3): 448-457.

AOAC (1980). Association of Official Agriculture Chemists 'Official Methods of Analysis', $13^{\text {rd }}$ Ed., Washington, D.C.

Bewley, J.D. and M. Black (1994). Seeds: Physiology of Development and Germination, Plenum Press, New York.

Bradford, K.J. (2002). Application of hydrothermal time to quantifying and modeling seed germination and dormancy. Weed Science, 50, 248-260.

Castelfranco, P.A. and S.I. Beale (1983). Chlorophyll biosynthesis : recent advances and area of current interest. Ann. Rev. Pl. Physiol., 34: 241-278.
Cuddeford, D. (1989). Hydroponic grass in practice, 11 (5): 211-214.

Draper, N.R. and H. Smith (1966). Applied Regression Analysis. illus. John Wiley and Sons, Inc., New York, 407.

El-Saidy, A.E.A. and K.M. Abd-El-Hai (2011). Alleviation of peanut seed deterioration during storage using biotic and abiotic agents. Res. J. Seed Sci., 4 (2): 68-81.

El-Tohamy, W.A. and N.H.M. El-Greadly (2007). Physiological responses, growth, yield and quality of snap beans in response to foliar application of yeast, vitamin $\mathrm{E}$ and zinc under sandy soil conditions. Aust. J. Basic and Appl. Sci., 1(3): 294-299.

Fazaeli, H., H.A. Golmohammadi, S.N. Tabatabayee and M. Asghari-Tabrizi (2012). Productivity and nutritive value of barley green fodder yield in hydroponic system. World Appl. Sci. J., 16 (4): 531-539.

Finch-Savage, W.E. and G. Leubner-Metzger (2006). Seed dormancy and the control of germination. New Phytologist, 171. doi: 10. 1111/j.1469-8137.2006.01787.x. 
Flores, J. and O. Briones (2001). Plant life-form and germination in a Mexican inter-tropical desert: effects of soil water potential and temperature. J. Arid Environ., 47 (4): 485-497.

Gomez, K.A. and A.A. Gomez (1984). Statistical Producer for Agricultural Research $2^{\text {nd }}$ Ed., John Wiley and Sons.

ISTA (2004). International Rules for Seed Testing. Int. Seed Testing Ass. Zürich, Switzerland.

Khan, M.A., B. Gul and D.J. Weber (2002). Improving seed germination of Salicorni arubra (Chenopodiaceae) under saline conditions using germination-regulating chemicals. Western North Ame. Nat., 62 (1): 101-105.

Koehler, P., G. Hartman, H. Wierser and M. Rychilk (2007). Changes of folates ,dietary fiber and protein in wheat as affected by germination. J. Agric. Food Chem., 55 : 4678-4683.

Milbau, A., B.J. Graae, A. Shevtsova and I. Nijs (2009). Effects of a warmer climate on seed germination in the subarctic. Ann. Bot., 104. doi: 10.1093/aob/mcp117.

Nejad, M.E., A. Bakhshandeh, M.H. Gharineh and A. Soltani (2015). Quantification of barley emergence to temperature. Int. J. Agric. Innovations and Res., 3: 2319-1473.
Rood, S.B., R.I. Buzzell, D.J. Major and R.P. Pharis (1990). Gibberellins and heterosis in maize: quantitative relationship. Crop Sci., 30: 281-6.

Saker, M.T., H.M. Ibrahim, M.I. Atta and M.A. Abd El-Aal (2015). Ascorbic and salicylic acids as well as seaweed and yeast extracts altered stress-related metabolites and enhanced yield and its quality of saltstressed soybean (Glycine $\max$ L.). J. Pl. Prod., Mansoura, 6 (9): 1459-1474.

Spencer, T.F.T., S.M. Dorothy and A.R.W. Smith (1983). Yeast genetics, fundamental and applied aspects, Springer. Verlag. New Yourk, USA, 16- 18, ISBNo-387-390973-9.

Tlig, T., M. Gorai and M. Neffati (2008). Germination responses of Diplotaxis harra to temperature and salinity. Flora-Morphol., Distribution, Functional Ecol. Pl., 203: 421428.

Van Soest, P.J., J.B. Robertson and B.A. Lewis (1991). Methods for dietary fiber, neutral detergent fiber and non-starch polysaccharides in relation to animal nutrition. J. Dairy Sci., 74 : 3583-3597.

Yousof, F.I., K.A. Amer and I.F. Mersal (2017). Screening of some Egyptian barley cultivars for sprouted green fodder yield. Int. J. Tech. Res. and Sci., 1 : 364-371. 


\section{بعض العوامل المؤثرة على إنتاج ونوعية محصول الثعير المستتبت بغرض العلف الأخضر}

\section{فيصل إبراهيم يوسف ـ الشيماء السيا إبراهيم مصطفى ـ مجاهد أحمد حلمي مجاهد}

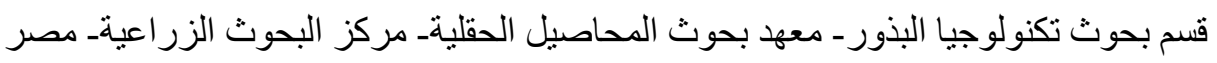

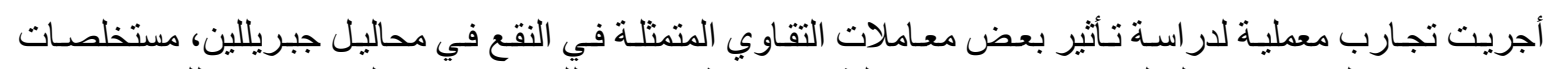

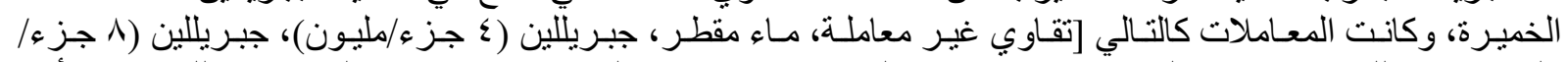

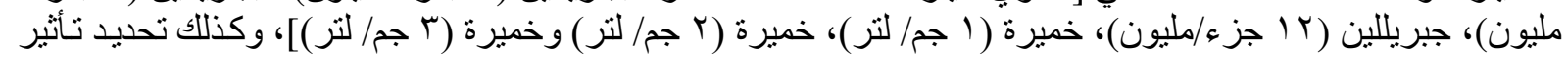

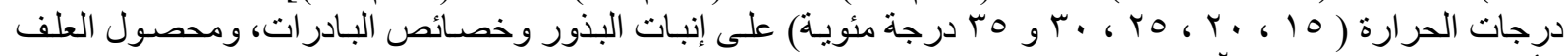

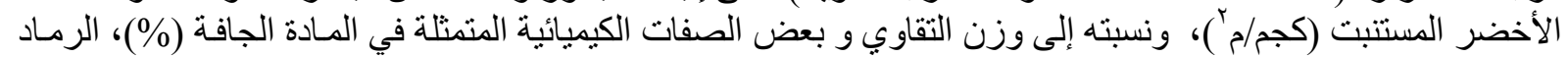

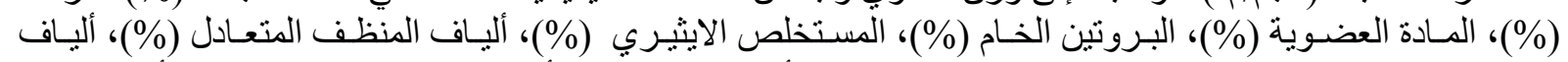

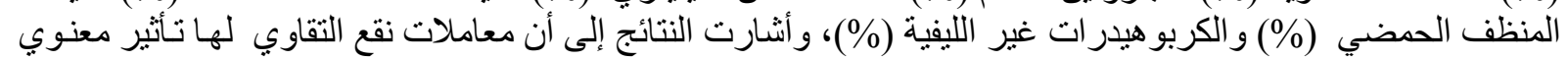

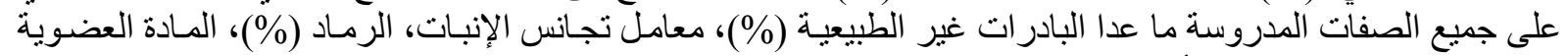

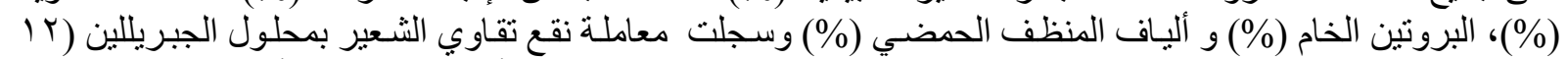

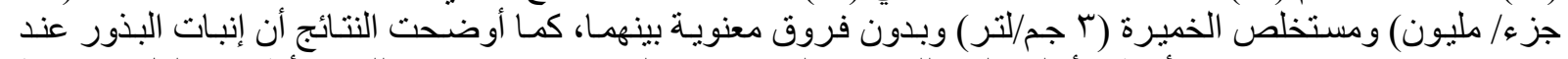

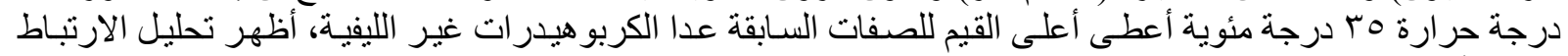

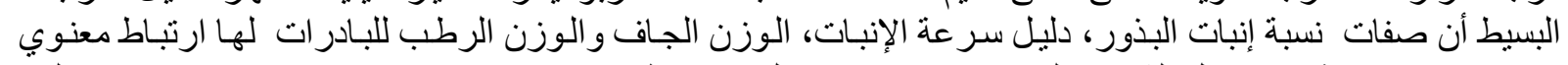

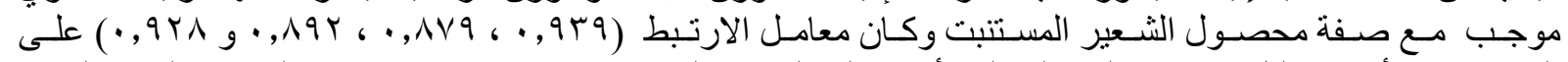

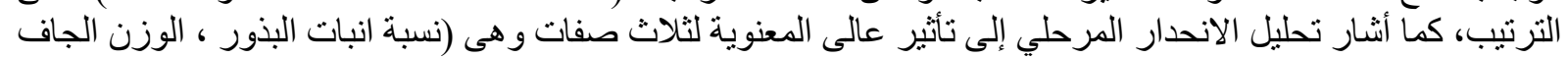

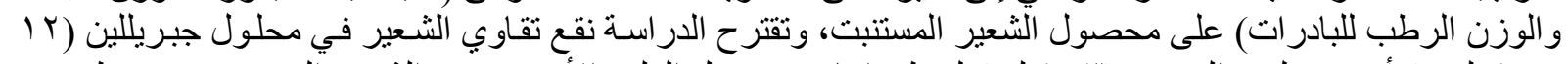

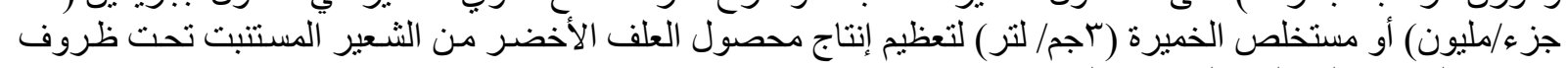
درجات الحرارة المختلفة على مدار العام. 